\title{
PGC1a promotes cholangiocarcinoma metastasis by upregulating PDHA1 and MPC1 expression to reverse the Warburg effect
}

Dan $\mathrm{Li}^{1}$, Chaoqun Wang ${ }^{1}$, Panfei Ma', Qingan $\mathrm{Yu}^{1}$, Mingqi Gu${ }^{1}$, Liqian Dong ${ }^{1}$, Wenjing Jiang ${ }^{1}$, Shangha Pan²,

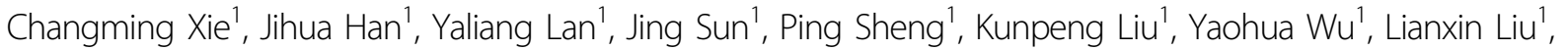
Yong $\mathrm{Ma}^{1}$ and Hongchi Jiang ${ }^{1,2}$

\begin{abstract}
PGC1a acts as a central regulator of mitochondrial metabolism, whose role in cancer progression has been highlighted but remains largely undefined. Especially, it is completely unknown about the effect of PGC1 a on cholangiocarcinoma (CCA). Here we showed that PGCla overexpression had no impact on CCA growth despite the decreased expression of PGC1a in CCA compared with adjacent normal tissue. Instead, PGCla overexpression-promoted CCA metastasis both in vitro and in vivo. Mechanistically, for the first time, we illuminated that PGC1a reversed the Warburg effect by upregulating the expression of pyruvate dehydrogenase E1 alpha 1 subunit and mitochondrial pyruvate carrier 1 to increase pyruvate flux into the mitochondria for oxidation, whereas simultaneously promoting mitochondrial biogenesis and fusion to mediate the metabolic switch to oxidative phosphorylation. On the one hand, enhanced mitochondrial oxidation metabolism correlated with elevated reactive oxygen species (ROS) production; on the other hand, increased PGC1 a expression upregulated the expression levels of mRNA for several ROS-detoxifying enzymes. To this end, the ROS levels, which were elevated but below a critical threshold, did not inhibit CCA cells proliferation. And the moderately increased ROS facilitated metastatic dissemination of CCA cells, which can be abrogated by antioxidants. Our study suggests the potential utility of developing the PGC1a-targeted therapies or blocking PGC1a signaling axis for inhibiting CCA metastasis.
\end{abstract}

\section{Introduction}

Recently, the overall incidence of cholangiocarcinoma (CCA) appears to have increased ${ }^{1,2}$, and cumulative CCA mortality has risen by $39 \%$ because of increased disease incidence ${ }^{3}$. The difficulty in diagnosing CCA in the early

\footnotetext{
Correspondence: Yong Ma (doctormy80@163.com) or Hongchi Jiang (jianghc2013@163.com)

'Department of Hepatic Surgery, The First Affiliated Hospital of Harbin Medical University, Key Laboratory of Hepatosplenic Surgery, Ministry of Education, Harbin, Heilongjiang Province, China

${ }^{2}$ Key Laboratory of Hepatosplenic Surgery, Ministry of Education, The First Affiliated Hospital of Harbin Medical University, Harbin, Heilongjiang Province, China

Edited by A. Finazzi-Agrò
}

stage, the low rate of surgical resection and its therapeutic resistance all contribute to its unfavorable prognosis. Therefore, there is an urgent need to understand the molecular mechanism underlying CCA progression.

Currently, metabolic reprogramming is now firmly established as "Achilles' heel" of cancer cells ${ }^{4}$. Tumors are even regarded as a metabolic disease ${ }^{5}$. The best characterized energy metabolism modification in cancer cells is the Warburg effect, wherein tumor cells oxidize a reduced fraction of the pyruvate generated from glycolysis. It is the long-term correlation of cancer metabolism with aerobic glycolysis that strikingly belies the crucial role of mitochondria in cancer cells ${ }^{6}$. Contrary to Otto

\section{(c) The Author(s) 2018}

(c) (i) Open Access This article is licensed under a Creative Commons Attribution 4.0 International License, which permits use, sharing, adaptation, distribution and reproduction c. in any medium or format, as long as you give appropriate credit to the original author(s) and the source, provide a link to the Creative Commons license, and indicate if changes were made. The images or other third party material in this article are included in the article's Creative Commons license, unless indicated otherwise in a credit line to the material. If material is not included in the article's Creative Commons license and your intended use is not permitted by statutory regulation or exceeds the permitted use, you will need to obtain permission directly from the copyright holder. To view a copy of this license, visit http://creativecommons.org/licenses/by/4.0/. 
Warburg's hypothesis that mitochondrial function in tumor cells is irreversibly damaged, it has been firmly demonstrated that mitochondria-mediated function is absolutely essential for tumor cells ${ }^{7}$. However, the exact functions of mitochondrial metabolism in cancer cells and the underlying molecular mechanisms remain poorly documented.

The peroxisome proliferator-activated receptor $\gamma$ coactivator-1 $\alpha$ (PPARGC1A, named hereafter PGC1 $\alpha$ ) is a member of the PGC1 family of coactivators ${ }^{8}$. PGC1 $\alpha$ acts as one of the central transcriptional regulators of gene networks involved in mitochondrial biogenesis and respiration ${ }^{9}$. The mitochondrial electron transport chain (ETC), especially complex I and III, is the main producer of reactive oxygen species (ROS); ${ }^{10}$ therefore, the increased mitochondrial content and enhanced mitochondrial respiratory activity stimulated by PGC1 $\alpha$ could in principle lead to an increase in the generation of ROS. Concurrently, PGC1 $\alpha$ is known to be able to potently upregulate ROS-detoxifying enzymes including glutathione peroxidase and manganese SOD to protect cells against oxidative stress ${ }^{11}$.

To date, it is incompletely understood what effect this seemingly paradoxical regulation of PGC1 $\alpha$ on ROS metabolism would have on the redox status of CCA. It is also unknown how PGC1 $\alpha$ regulates glucose metabolism and ROS metabolism in CCA and what are the growth and metastasis consequences this might cause to CCA.

\section{Results}

PGC1 a expression was downregulated in CCA tissues and cell lines

The qRT-PCR results indicated that the PGC1 $\alpha$ mRNA levels were highly suppressed in CCA tissues compared with normal bile duct samples (Fig. 1a). PGC1 $\alpha$ protein levels were verified to be markedly lower in CCA samples than in nonmalignant counterparts by western blot (Fig. 1b). Accordingly, the immunohistochemistry (IHC) results showed that PGC1 $\alpha$ exhibited weak or negative staining in CCA samples with a rate of $69 \%(69 / 100)$, whereas in normal bile duct tissues the rate of weak or negative PGC1 $\alpha$ staining was only $16.7 \% \quad(5 / 30) \quad$ (Fig. 1c). Regarding different cell lines, the PGC1 $\alpha$ mRNA and protein levels detected in HIBEpiC cells, the normal human intrahepatic biliary epithelial cell line, were higher than those in five CCA cell lines (Fig. 1d,e). Subsequently, three CCA cell lines with very high levels (CCLP1), moderate levels (HCCC-9810) or very low levels (HuCCT1) of PGC1 $\alpha$ mRNA and protein expression were chosen for further experiments. Together, all these results demonstrate that PGC1 $\alpha$ levels are reduced in CCA tissues and cell lines.

\section{PGC1a has no impact on CCA proliferation but promotes CCA metastasis}

To assess the role of PGC1 $\alpha$ in CCA cells proliferation, we transfected PGC1 $\alpha$-expressing lentiviral vectors or lentiviral-based shRNA against PGC1 $\alpha(\operatorname{shPGC1} \alpha)$ into CCA cells. Both the overexpression and knockdown of PGC1 $\alpha$ were confirmed by qRT-PCR and western blot (Supplementary Figure 1). Cell growth assays showed that PGC1 $\alpha$ overexpression and knockdown did not affect the viability of the indicated CCA cells over the course of 7 days (Fig. 2a). Accordingly, PGC1 $\alpha$ did not affect colony number, cell apoptosis, cell cycle progression or cellular senescence (Fig. 2b-e) in CCA cells. Consistent with our in vitro data, PGC1 $\alpha$-transduced HuCCT1 tumors showed similar tumor growth kinetics and weight compared with HuCCT1-control tumors, and tumor size did not differ significantly between PGC1 $\alpha$-depleted cells and shScrbl cells (Fig. 2f).

PGC1 $\alpha$ has been reported to be involved in tumor metastasis without having effect on tumor growth ${ }^{12}$. Therefore, we next examined the effect of PGC1 $\alpha$ on metastasis of CCA cells. Surprisingly, overexpression of PGC1 $\alpha$ significantly increased the migratory and invasive capabilities of HuCCT1 and HCCC-9810 cells, and suppression of PGC1 $\alpha$ diminished the migratory and invasive properties of CCLP1 and HCCC-9810 cells, as assessed by migration assays and wound-healing assays and invasion assays (Fig. 3a, b and Supplementary Figure 2A). In addition, as for CCLP1, HCCC-9810 and HuCCT1 cell lines, cells with higher endogenous levels of PGC1 $\alpha$ displayed higher invasive capability (Supplementary Figure 2B). Furthermore, Higher anchorage-independent survival rates in cells overexpressing PGC1 $\alpha$ was observed compared to control cells, and the percent of live cells was lower in shPGC1 $\alpha$ cells than in shScrbl cells, assessed by anoikis assays (Fig. 3c).

Consistent with the in vitro data, the results of the peritoneal cavity metastasis models revealed that HuCCT1-PGC1 $\alpha$ cells formed more metastatic nodules than HuCCT1-control cells, whereas the number of metastatic nodules was fewer in mice harboring CCLP1-shPGC1 $\alpha$ cells than that in the control group (Fig. 3d). Correspondingly, the results of the lung metastasis models also showed that the volume of lung metastases were significantly increased in mice bearing HuCCT1-PGC1 $\alpha$ cells than that in the control group, whereas mice implanted with CCLP1-shPGC1 $\alpha$ cells had smaller lung metastases compared with the control group (Fig. 3e and Supplementary Figure 2C). Collectively, these results demonstrate that PGC1 $\alpha$ affects neither CCA cells proliferation in vitro nor the primary tumor growth kinetics in vivo but does significantly promote CCA cells migration and invasiveness, both in vitro and in vivo. 

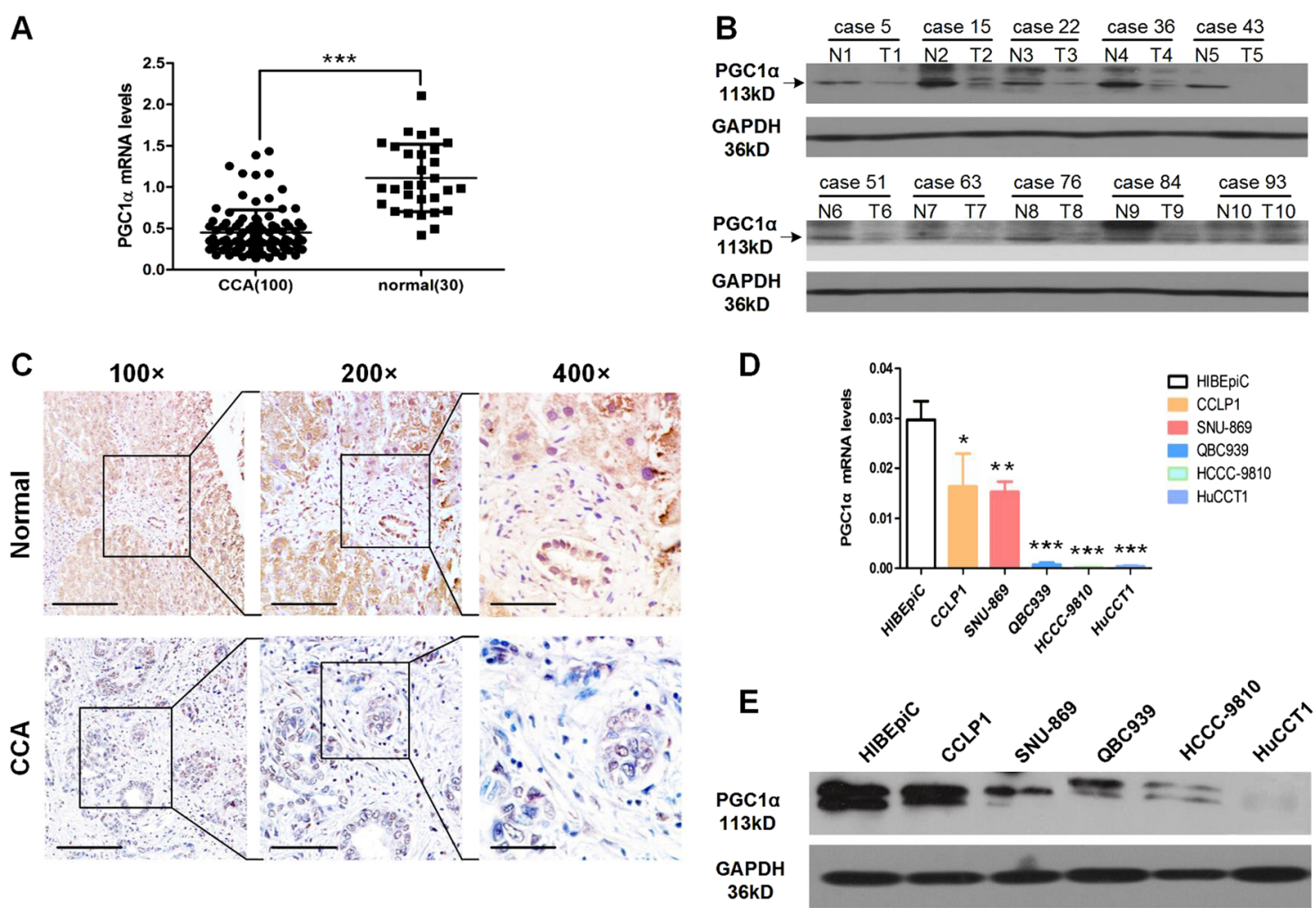

Fig. 1 PGC1a is downregulated in human CCA tissues and cell lines. The samples were from our patients. a PGC1a mRNA levels in CCA tissues or normal bile duct tissues. $\mathbf{b}$ PGC1 a protein levels in CCA tissues or adjacent nontransformed tissues. T: tumors; N: adjacent nontransformed tissues. c Representative images of PGC1a IHC staining in normal bile duct tissues and CCA tissues. Scale bars, $\times 40: 1 \mathrm{~mm} ; \times 100: 400 \mu \mathrm{m} ; \times 400: 100 \mu \mathrm{m}$. d Relative PGC1 a mRNA levels in HIBEpiC and five CCA cell lines by qRT-PCR. e PGC1a protein levels in HIBEpiC and 5 CCA cell lines by western blot. All bar graphs are plotted as mean \pm SD of three independent experiments performed in triplicate. ${ }^{*} P<0.05,{ }^{* *} P<0.01,{ }^{* * *} P<0.001$

Given the counterintuitive results, we next assessed the effect of PGC1 $\alpha$ expression on clinical cases of CCA. The CCA patients were segregated into two or three groups based on the IHC results: a PGC1 $\alpha$-high group and a PGC1 $\alpha$-low or (and) -negative group. Surprisingly, there was a significant correlation between high PGC1 $\alpha$ expression and a worse overall survival rate. Accordingly, patients whose tumors exhibited relatively high expression of PGC1 $\alpha$ were significantly more likely to have advanced-stage tumors and metastatic disease (Fig. 3f). These results illustrate that relatively high PGC1 $\alpha$ expression positively correlates with poor prognosis in CCA patients.

\section{PGC1a enhances pyruvate oxidation metabolism in CCA cells}

We next explored the transcriptional program associated with the metastasis promoting activity of PGC1 $\alpha$. Gene expression profiling coupled with bioinformatic analyses revealed that the metabolic pathways were the most differentially modulated canonical pathways in HuCCT1-PGC1 $\alpha$ cells compared with HuCCT1-control cells. Notably, among the top 20 significantly altered pathways, most were related to mitochondrial metabolism, including pyruvate metabolism, the TCA cycle and oxidative phosphorylation (OXPHOS) (Fig. 4a). The heatmap unveiled a significant increase in transcription levels related to mitochondrial metabolism in HuCCT1PGC1 $\alpha$ cells. Interestingly, two key genes related to pyruvate metabolism reached a significant increase $(>1.5$ fold), including pyruvate dehydrogenase E1 alpha 1 subunit (PDHA1) and mitochondrial pyruvate carrier 1 (MPC1) (Fig. 4b). PDHA1 encodes the E1 alpha 1 subunit of PDH complex, which contains the E1 active sites and has a critical role in the function of PDH complex. MPC1 is a gate-keeping mitochondrial protein that control the entry of pyruvate into the mitochondria. Therefore, we speculated that PGC1 $\alpha$ could reverse the Warburg effect by upregulating the expression of PDHA1 and $\mathrm{MPC} 1$ and simultaneously promote gene expression of the TCA and OXPHOS, thus enhancing mitochondrial metabolism.

In support of our hypothesis, by analyzing gene expression in CCA samples from The Cancer Genome Atlas data sets, we found that the expression levels of PDHA1 and MPC1 were positively correlated with 


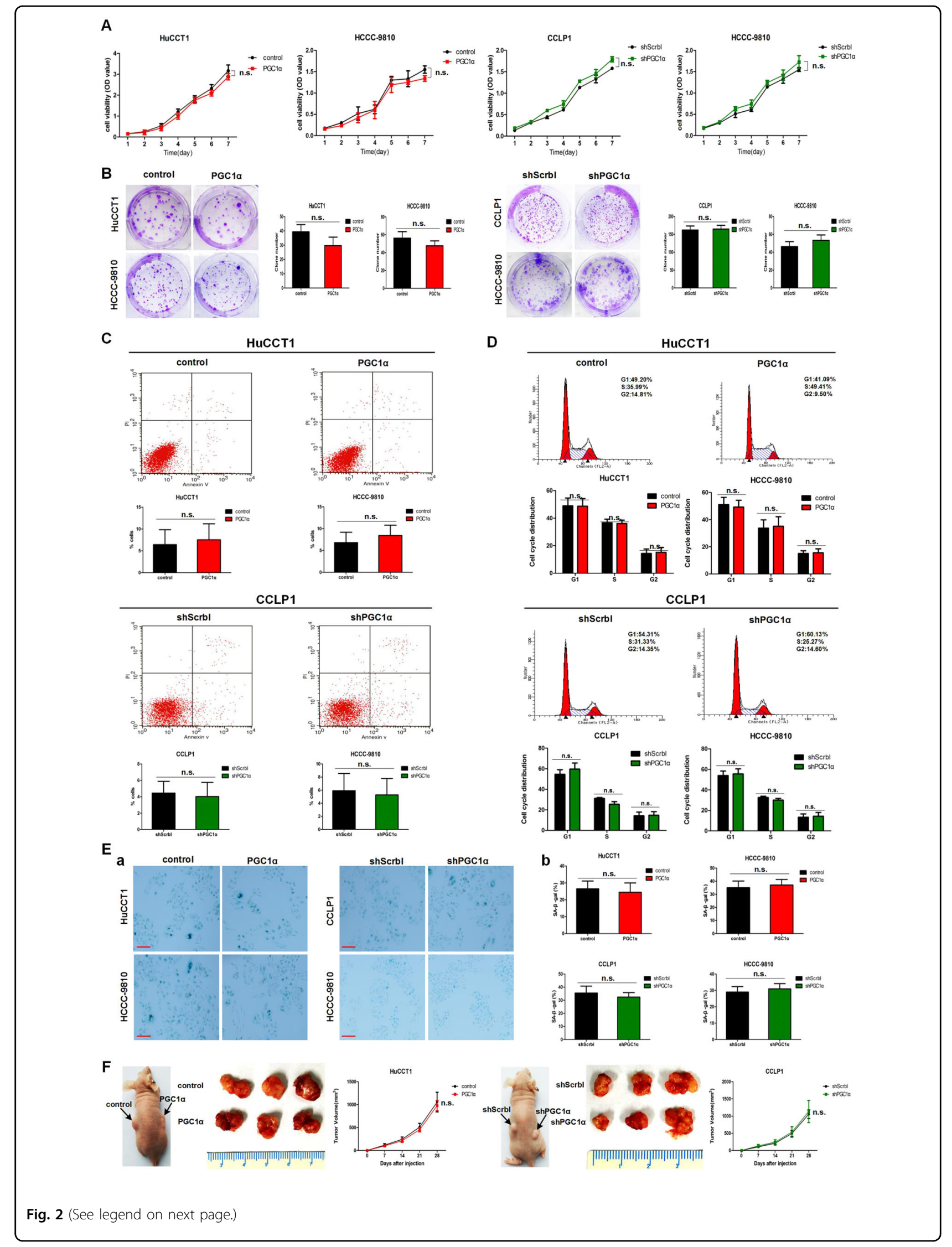


(see figure on previous page)

Fig. 2 PGC1a has no effect on CCA cell proliferation and tumorigenesis. a Growth curves for the indicated CCA cells evaluated by the CCK-8 assays. b Representative images of the colony formation assays and analysis results. c Cell apoptosis determined by Annexin V/PI staining. $\mathbf{d}$ Representative images of the cell cycle analysis and analysis results of indicated CCA cells. e a Representative images of SA- $\beta$-gal staining in the indicated cells. Scale bars: $100 \mu \mathrm{m}$. b The quantification of positively SA- $\beta$-gal staining cells. f Tumor growth of HuCCT1 $(n=10)$ and CCLP1 ( $n=10)$ xenografts and representative xenograft tumor images. The tumor growth curves are plotted as mean \pm SD ( $n=10$, each group). All line graphs and bar graphs are presented as mean \pm SD of three independent experiments performed in triplicate except otherwise stated. n.s. represents no statistical significance

PGC1 $\alpha$ in CCA samples (Supplementary Figure 3). Furthermore, in clinical samples, $80.65 \%$ (25 cases) and $70.97 \%$ (22 cases) of samples with high PGC1 $\alpha$ expression (31 cases) exhibited high level of PDHA1 and MPC1 respectively, whereas $75.36 \% \quad(52$ cases $)$ and $84.06 \%$ (58 cases) of samples with low/negative PGC1 $\alpha$ expression (69 cases) showed low expression of PDHA1 and MPC1 respectively (Fig. 4c). The mRNA and protein levels of PDHA1 and MPC1 were elevated following PGC1 $\alpha$ overexpression (Fig. 4d,e). Accordingly, $\mathrm{PDH}$ enzyme activity was increased in PGC1 $\alpha$ overexpressing cells compared with control cells (Fig. 4f).

\section{PDHA1 and MPC1 are direct transcriptional targets of PGC1a/NRF1}

Next, we hypothesized that PGC1 $\alpha$ transactivates PDHA1 and MPC1 gene transcription by binding to a transcription factor (TF). We analyzed the PDHA1 and MPC1 promoter sequences using the JASPAR, PROMO and SWISSREGULON algorithms and found that NRF1 is a common TF. More importantly, NRF1 has been linked to the transcriptional control of many genes involved in mitochondrial function and biogenesis ${ }^{13}$. Therefore, we speculated that the TF most likely to interact with PGC1 $\alpha$ was NRF1. The interaction between PGC1 $\alpha$ and NRF1 was confirmed by co-immunoprecipitation (co-IP) assays (Fig. 5a). We found putative NRF1-binding sites on the PDHA1 or MPC1 promoter by the JASPAR algorithm. As shown in Fig. 5b, the PDHA1 and MPC1 NRF1 sites are near-perfect matches to the NRF1 consensus. Electrophoresis mobility shift assays (EMSA) indicated that DNA-protein complexes were formed using crude nuclear extracts of HuCCT1-PGC1 $\alpha$ cells and radiolabeled PDHA1 and MPC1 NRF1 oligomers (Fig. 5c). Next, the candidate binding sites of the promoters were cloned at $5^{\prime}$ of the SV40 promoter and fused to the luciferase reporter. To the end, PGC1 $\alpha$ and NRF1 induced a significant increase in the activity of luciferase reporter carrying PDHA1 or MPC1 promoter fragment (Fig. 5d). Accordingly, chromatin immunoprecipitation (ChIP) assays showed that the promoter regions of PDHA1 and MPC1 were amplified from the DNA recovered from the immunoprecipitation complex using
NRF1 antibody (Fig. 5e). Taken together, we first demonstrate that PGC1 $\alpha / \mathrm{NRF} 1$ complexes can directly bind to the PDHA1 and MPC1 promoters to promote gene transcription.

PGC1a expression reverses the Warburg effect, mediating the metabolic switch from aerobic glycolysis to OXPHOS

Liquid chromatography-mass spectrometry (LC-MS)/ MS metabolomics validated that HuCCT1-PGC1 $\alpha$ cells had increased levels of TCA intermediates (Fig. 6a). Subsequent biochemical assays also confirmed the decreased glucose uptake and decreased lactate production in PGC1 $\alpha$-transduced cells (Fig. 6b). We then measured the energy phenotype of the cells. PGC1 $\alpha$ overexpressing CCA cells exhibited substantially increased basal and maximal oxygen consumption rates (OCR) (Fig. 6c). We also observed a decrease in extracellular acidification rate (ECAR) in PGC1 $\alpha$ overexpression CCA cells (Supplementary Figure 4A). Consistent with oxidative metabolism generating higher ATP levels than glycolytic metabolism, the intracellular ATP levels in PGC1 $\alpha$-overexpressing CCA cells were increased (Fig. 6d).

Conversely, depletion of PGC1 $\alpha$ levels appears to be critical for enabling and promoting the Warburg effect in CCA cells, e.g., decreased basal OCR, increased ECAR, increased glucose uptake and lactate production, and reduced intracellular ATP levels (Supplementary Figure 4B-4F).

Furthermore, we explored whether the PGC1 $\alpha$ overexpression-promoted CCA metastasis could be reversed by knockdown of PDHA1 and MPC1 in CCA cell lines. The silencing efficiency by siRNA was confirmed by qRT-PCR (Supplementary Figure 5A). We found that knockdown of PDHA1 and MPC1 reversed the avidity of PGC1 $\alpha$ overexpression cells for OXPHOS (Fig. 6e) and blocked tumor cells migration and invasion mediated by PGC1 $\alpha$ (Fig. $6 f$ and Supplementary Figure 5B).

In aggregate, our results support that $\mathrm{PGC1} \alpha$ reverses the Warburg effect by upregulating PDHA1 and MPC1 expression, thus enhancing mitochondrial metabolism and facilitating CCA cells migration and invasion. 


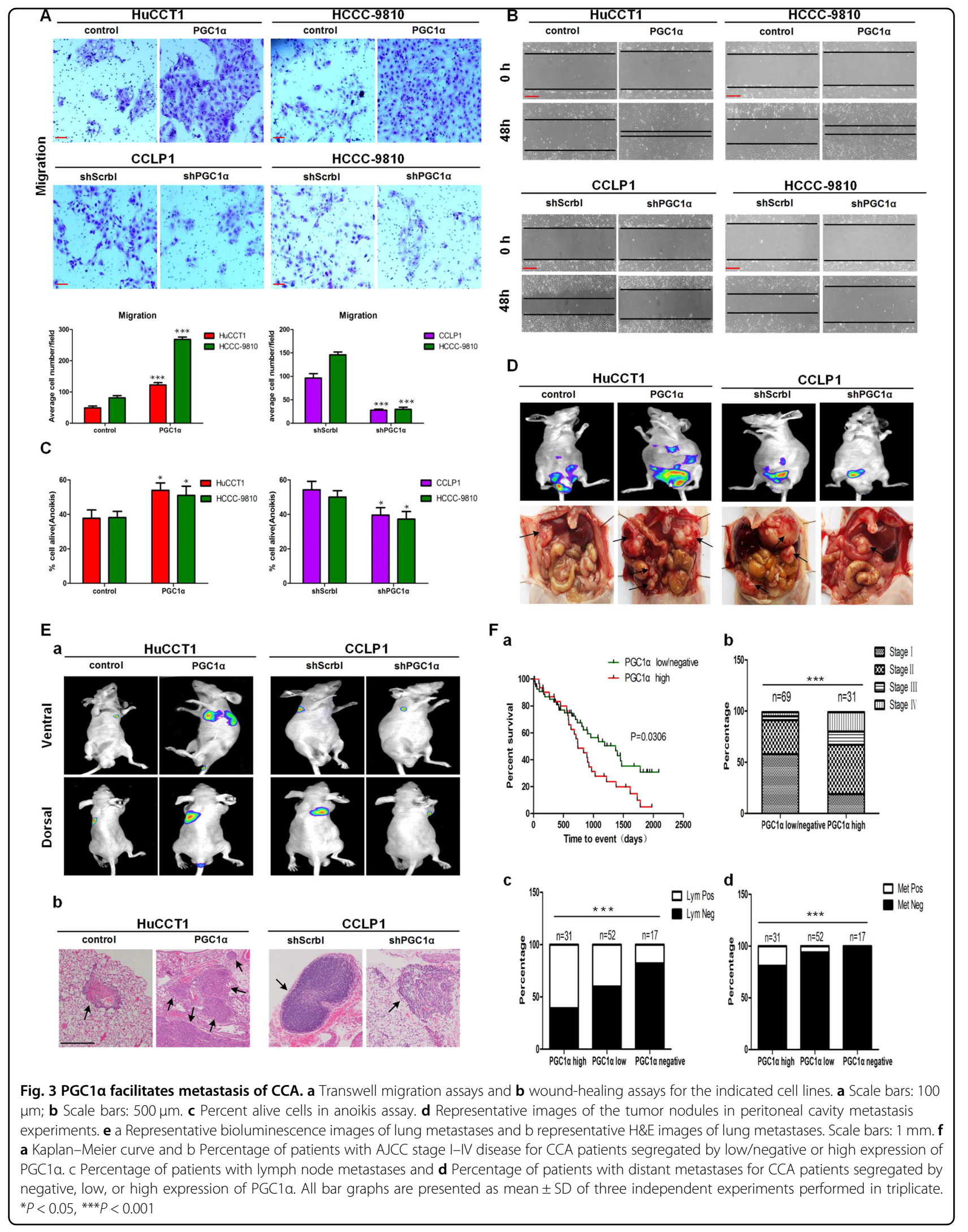




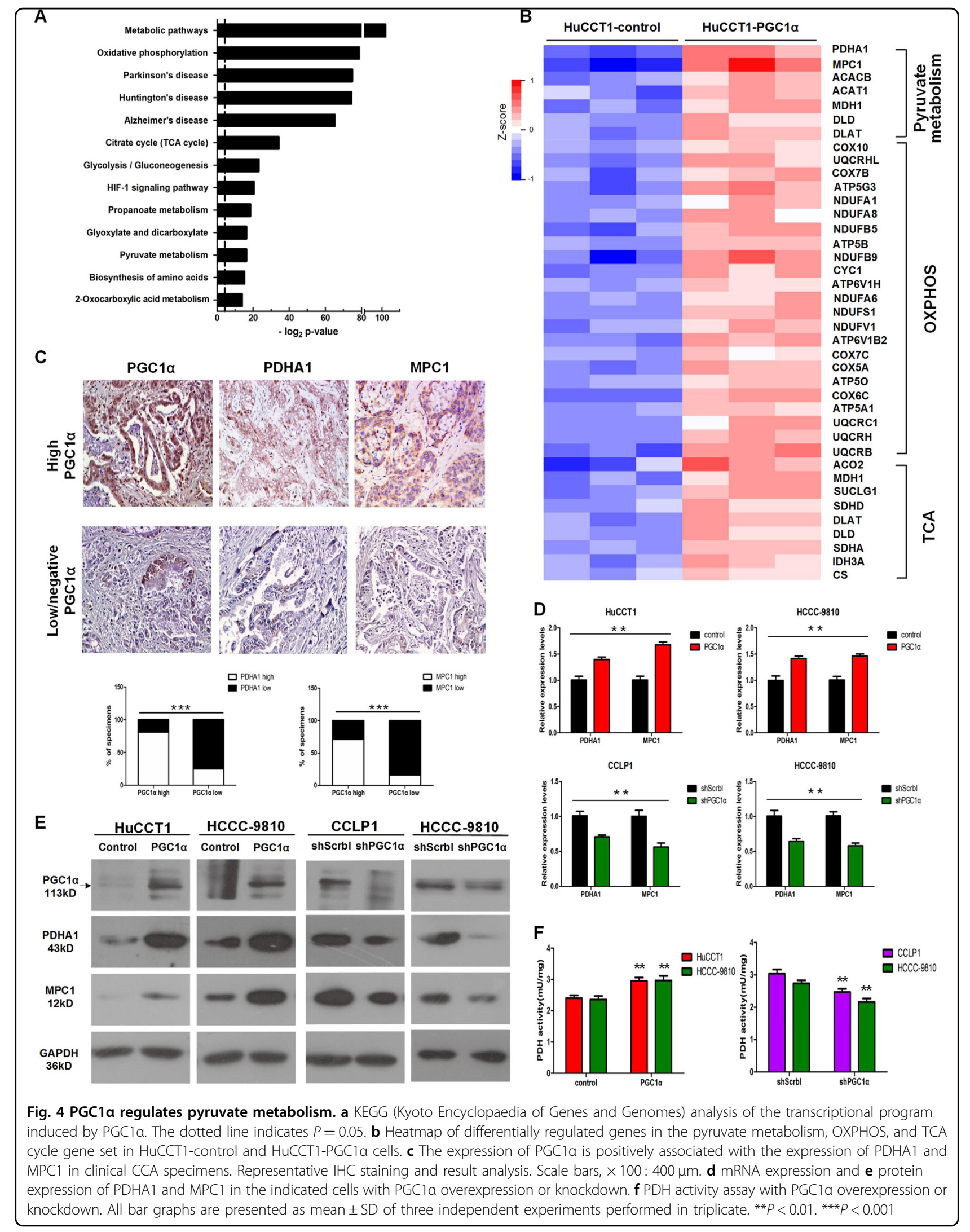


A

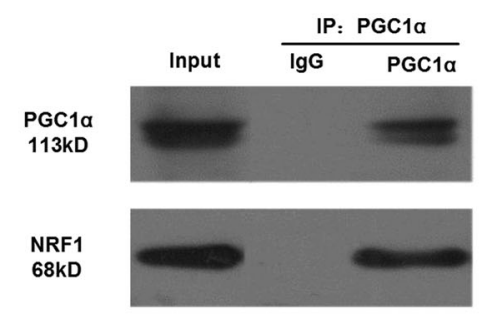

B

Y G C G C A Y G C G C R

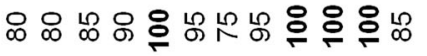

-168 C A C G C A G G C G C T -155

-120 C C C C C G T G C G C C -107
NRF-1 consensus

PDHA1 NRF1

MPC1 NRF1
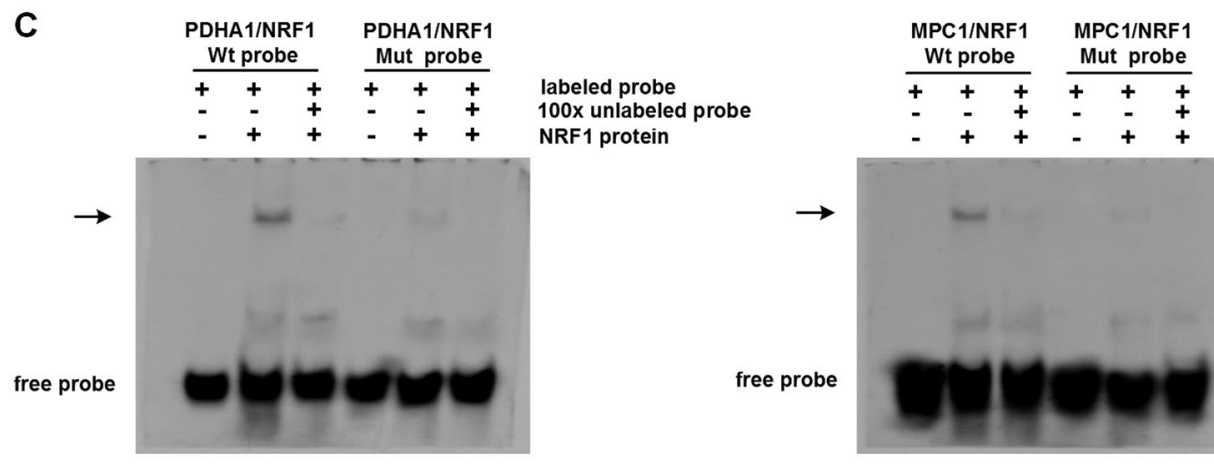
NRF1 protein

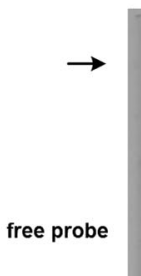

labeled probe 100x unlabeled probe NRF1 protein

D PDHA1 promoter luciferase

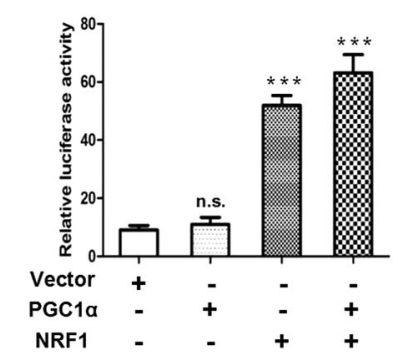

MPC1 promoter luciferase

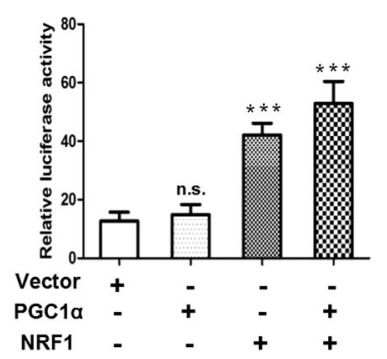

E

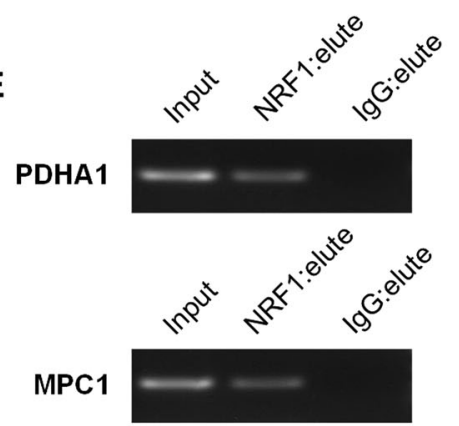

Fig. 5 PGC1a/NRF1 complexes directly bind to and activate PDHA1 and MPC1 promoters. a Results of the co-IP assays shows that PGC1a forms a complex with NRF1 in HuCCT1- PGC1 a cells. b NRF1 sites from PDHA1 and MPC1 are compared with the consensus. $\mathbf{c}$ EMSA were performed with nuclear extracts and radiolabeled probes encompassing the candidate NRF1-binding sequence on the PDHA1 or MPC1 promoter. $\mathbf{d}$ The effect of PGC1a and NRF1 on transcriptional activity of PDHA1 and MPC1 promoters, measured by luciferase reporter assays. Data are the mean \pm SD of three independent experiments performed in triplicate. e ChIP assay showing the binding of NRF1 to PDHA1 or MPC1 promoter in vivo. The promoter regions of PDHA1 or MPC1 were amplified using the input and immunoprecipitated DNA as templates. ${ }^{* * *} P<0.001$

\section{PGC1a promotes mitochondrial biogenesis and}

\section{mitochondrial fusion to direct the metabolic shift}

Corroborating microarray results and previous studies indicating that PGC1 $\alpha$ has a central role in regulating the TCA cycle and OXPHOS, genes predominantly related to the mitochondrial TCA cycle and OXPHOS were significantly upregulated in PGC1 $\alpha$-overexpressing cells (Fig. 7a). Accordingly, the mitochondrial DNA copy number was elevated in PGC1 $\alpha$-overexpressing CCA cells and decreased in PGC1 $\alpha$ knockdown CCA cells (Fig. 7b). Similarly, ectopic expression of PGC1 $\alpha$ increased the fluorescence intensity of MitoTracker, an indicator of mitochondrial mass, whereas PGC1 $\alpha$ knockdown cells exhibited weaker fluorescence intensity than shScrbl cells (Fig. 7c).
Mitochondria continually display a continuous cycle of fusion and fission, which has been linked to mitochondrial respiratory capacity ${ }^{14}$. Mitochondrial fusion is associated with high respiration capacity ${ }^{15}$. Considering that PGC1 $\alpha$ powerfully promotes mitochondrial respiration, we hypothesized that PGC1 $\alpha$ facilitated mitochondrial fusion to enhance OXPHOS. As expected, transmission electron microscopy showed that in addition to the increased number of mitochondria, mitochondrial volume was also significantly increased in PGC1 $\alpha$-overexpressing cells (Fig. 7d). Mitofusin-1(Mfn1), mitofusin-2 (Mfn2) isoforms, and optic atrophy 1 (OPA1) protein are three key proteins mediating mitochondrial fusion ${ }^{16}$. Interestingly, all three genes have previously been reported to be direct PGC1 $\alpha / E R R \alpha$ gene targets ${ }^{17}$. Indeed, the 


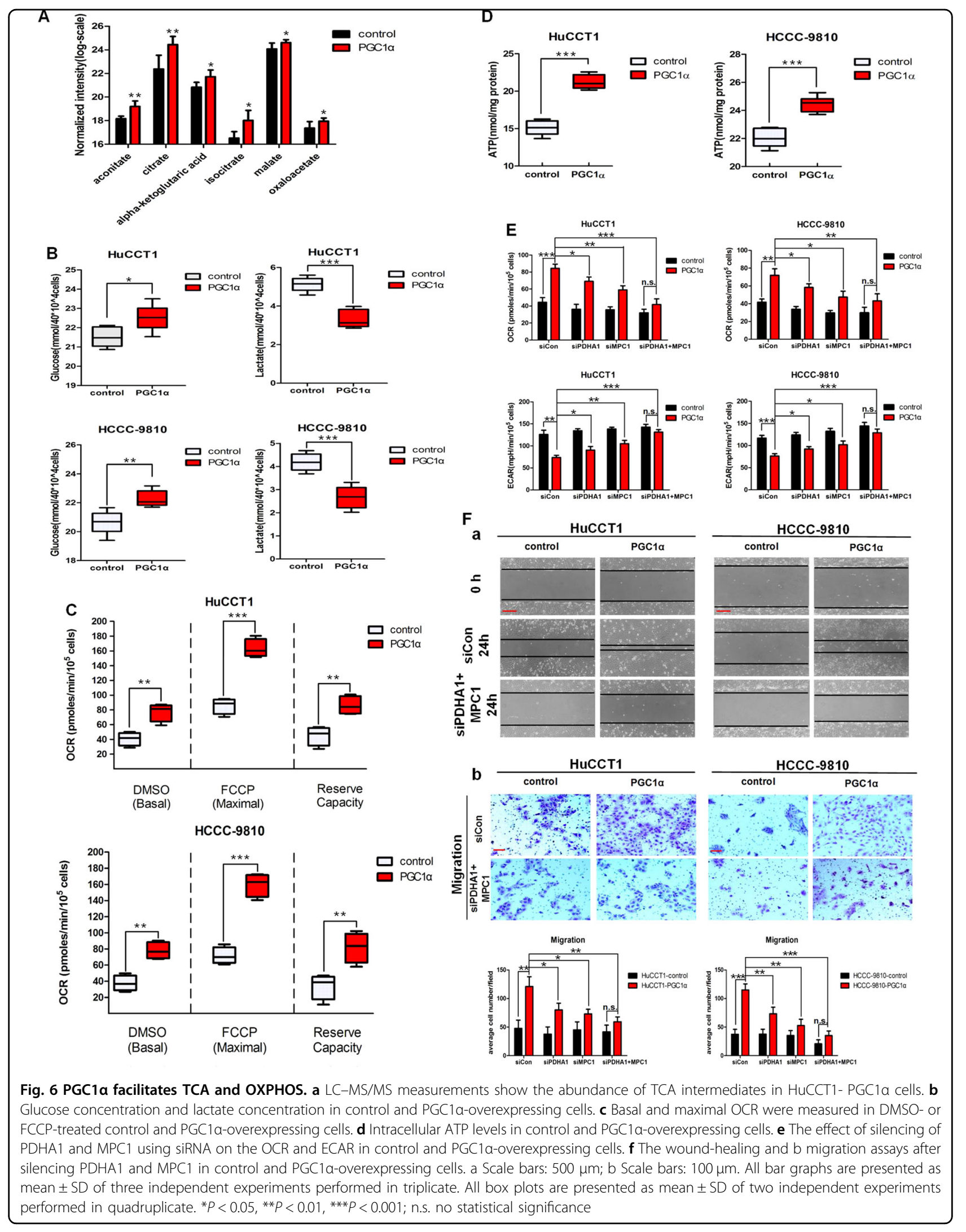




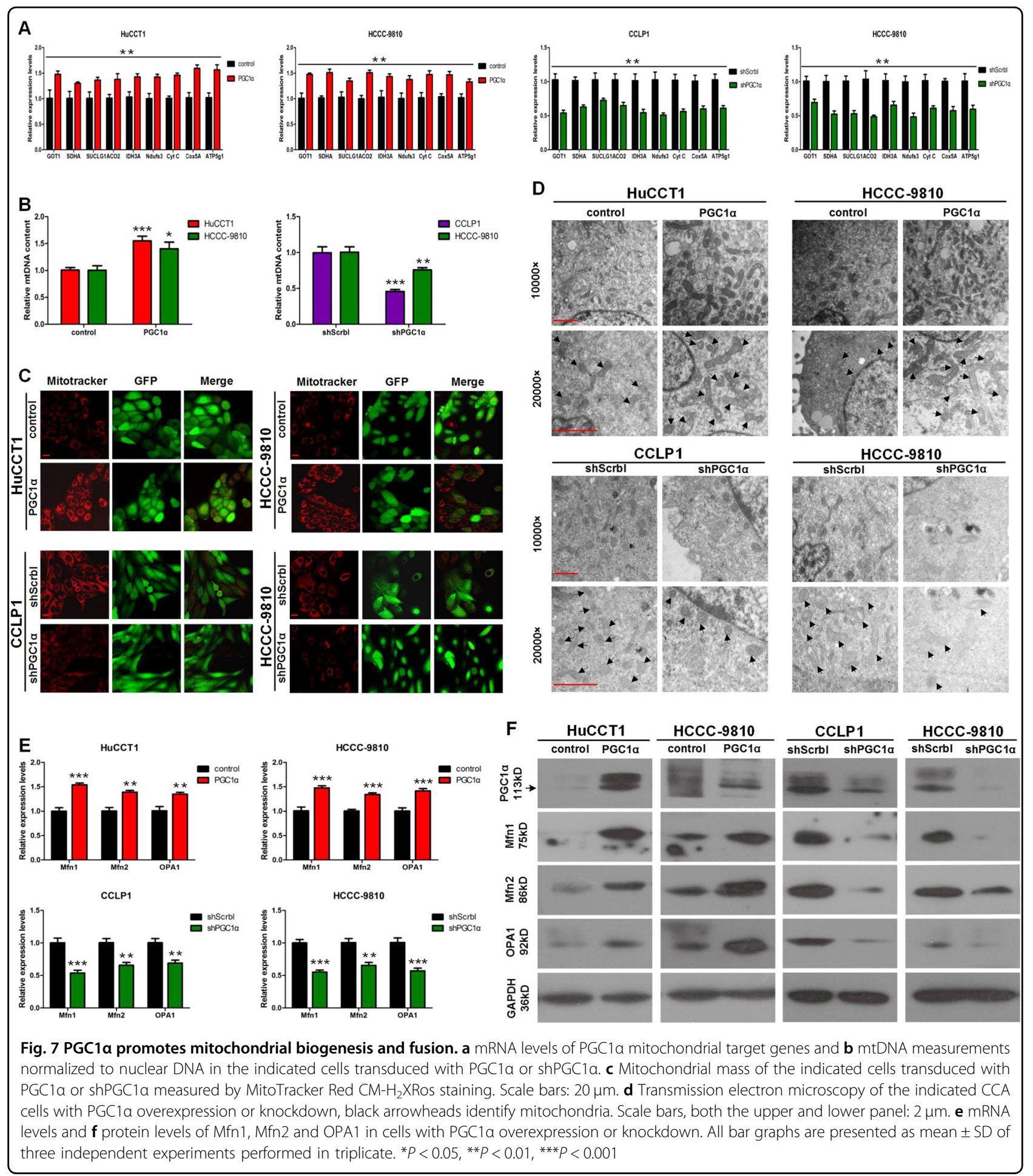

mRNA levels (Fig. 7e) and protein levels (Fig. 7f) of the three genes were significantly upregulated following the overexpression of PGC1 $\alpha$. Taken together, our findings converge to demonstrate that PGC1 $\alpha$ drives mitochondrial biogenesis and fusion to boost OXPHOS capacity.
PGC1a increases ROS production and promotes CCA metastasis

In view of the dual regulation of PGC1 $\alpha$ on ROS levels and the complex effect of ROS on cancer cells, we further investigated the role of ROS in the process of CCA metastasis induced by PGC1 $\alpha$. As reported previously, 


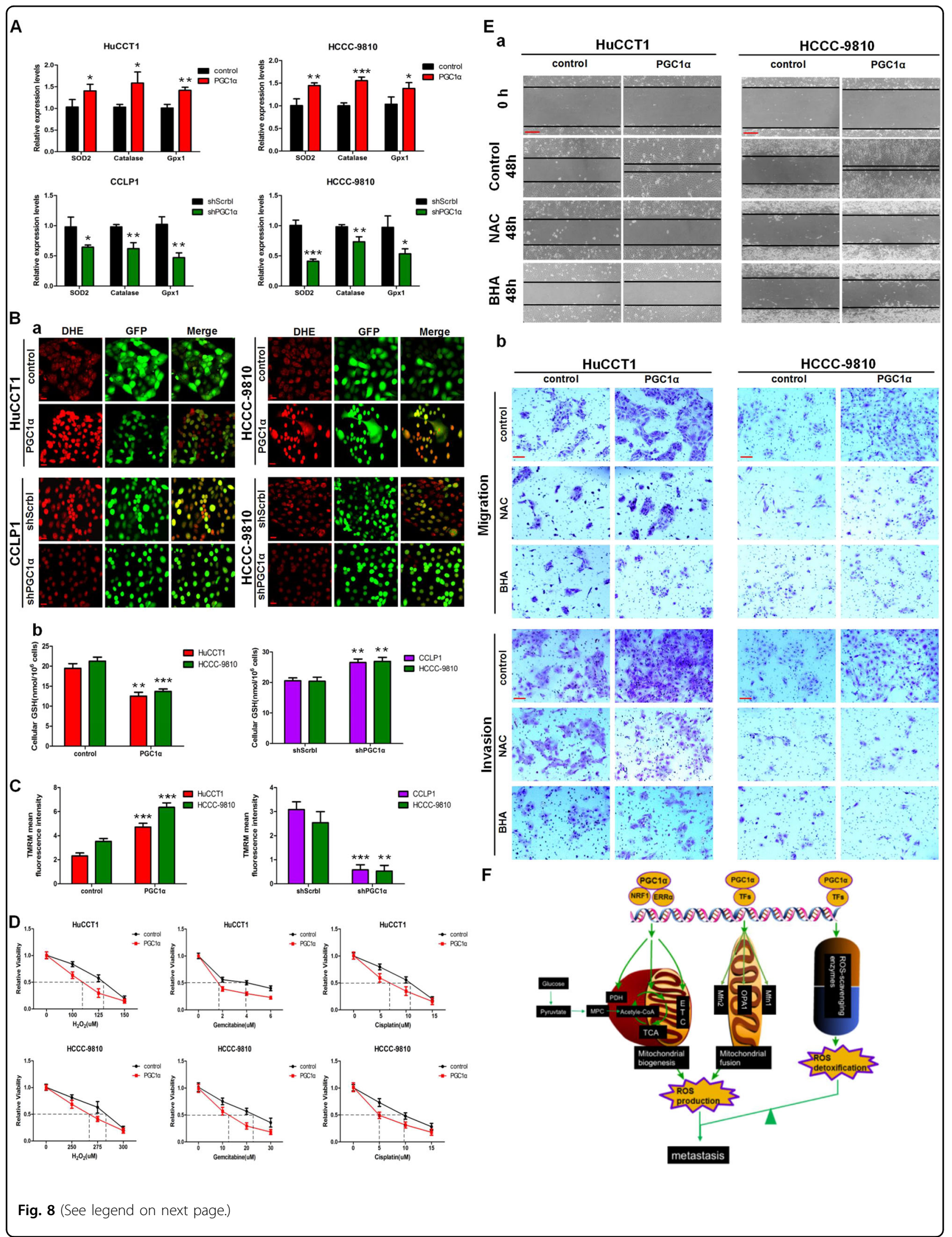


(see figure on previous page)

Fig. 8 PGC1a facilitates CCA metastasis but simultaneously enhances sensitivity to cytotoxic therapies by increasing ROS levels. a mRNA levels of ROS detoxification enzyme genes in PGC1a or shPGC1a-transduced cells. b a ROS levels, detected by DHE fluorescent dye and b cellular GSH levels after overexpression or depletion of PGC1a. Scale bars: $20 \mu \mathrm{m}$. c Mitochondrial membrane potential, measured by TMRM staining in PGC1a overexpression or knockdown cells. $\mathbf{d}$ CCK-8 assay of control and PGCla-transduced cells treated with the indicated dose of the indicated cytotoxic drugs for $48 \mathrm{~h}$. e Representative images of a Wound-healing assays and b migration and invasion assays of the PGC1a overexpressing cells treated with NAC or BHA. a Scale bars: $500 \mu \mathrm{m}$; b Scale bars: $100 \mu \mathrm{m}$. f Schematic presentation of the pathway underlying PGC1a-facilitated CCA metastasis. All bar graphs are presented as mean \pm SD of three independent experiments performed in triplicate. ${ }^{*} P<0.05,{ }^{* *} P<0.01,{ }^{* * *} P<0.001$

PGC1 $\alpha$ expression did increase the expression of several antioxidant genes (Fig. 8a). However, dihydroethidium staining showed that in PGC1 $\alpha$-overexpressing cells, intracellular concentrations of ROS were increased, accompanied by a decrease in GSH levels (Fig. 8b), indicating that the antioxidant response was not sufficient to detoxify the increase in ROS. It has been reported that increased ROS generation could decrease mitochondrial membrane potential $(\Delta \psi \mathrm{m})$ and sequentially trigger mitochondria-dependent apoptosis ${ }^{18}$. However, we did not observe a decrease of $\Delta \psi \mathrm{m}$ in PGC1 $\alpha$-overexpressing cells. Instead, we found a higher fluorescence intensity in PGC1 $\alpha$-overexpressing cells with the $\Delta \psi \mathrm{m}$-sensitivepositive dye tetramethylrhodamine methyl ester, reflecting increased ETC activity and mitochondrial function (Fig. 8c and Supplementary Figure 6A). In addition, we tested whether increased ROS levels would sensitize CCA cells to cytotoxic therapies. The results showed that increased ROS rendered PGC1 $\alpha$-overexpressing cells more sensitive to $\mathrm{H}_{2} \mathrm{O}_{2}$ and chemotherapeutic drugs gemcitabine and cisplatin (Fig. 8d). Subsequently, we investigated whether the antioxidants $N$-acetyl- $L$-cysteine (NAC) and butylated hydroxyanisole (BHA) could antagonize the metastatic phenotype of PGC1 $\alpha$. NAC and BHA abolished the increase in ROS levels (Supplementary Figure $6 \mathrm{~B}$ ) and blunted to varying extents the promotion effect of PGC1 $\alpha$ on migration and invasion in HuCCT1 and HCCC-9810 cells (Fig. 8e and Supplementary Figure 7). Taken together, our data indicate that PGC1 $\alpha$ leads to an increase in ROS levels and thus promotes CCA metastasis.

\section{Discussion}

Our data showed that the PGC1 $\alpha$ expression was reduced in CCA compared with noncancerous tissue. However, at odds with our expectations, PGC1 $\alpha$ overexpression indeed tended to inhibit CCA cell growth but that was not statistically significant, suggesting that PGC1 $\alpha$ possibly is not involved in growth signaling pathways. Intriguingly, we reveal that $\mathrm{PGC} 1 \alpha$ promotes CCA metastasis with solid evidence for the first time. With regard to why the expression of PGC1 $\alpha$ acting as an oncogene is suppressed in CCA, we speculate that PGC1 $\alpha$ underexpression just reflects damage of mitochondrial metabolism in CCA cells, for PGC1 $\alpha$ is an indicator for mitochondrial metabolism. The effect of enhanced mitochondrial metabolism on tumor growth is not clear ${ }^{12,19,20}$. However, most metastatic cancer cells depend on mitochondrial metabolism to produce energy ${ }^{21,22}$. Our data also support that CCA cells with relatively more active mitochondrial metabolism are more likely to metastasize. Possibly it also depends on which kind of cancer and which organ site cancer cells will colonize to, for instance, liver metastatic breast cancer cells showed increased glycolysis activity by activating PDK1 and thus inhibiting $\mathrm{PDH}^{23}$.

One major disadvantage of our study is that we find it difficult to demonstrate PGC1 $\alpha$ expression is higher in metastatic CCA than that in non-metastatic CCA. Our tissue samples are from patients with no detectable metastases preoperatively, whereas samples from those patients with distant metastasis are not available for there is no opportunity for surgery. Therefore, there might exist a selection bias related to sample collection and it is difficult to compare PGC1 $\alpha$ expression difference between matastatic CCA and non-metastatic CCA owing to the small number and lack of representation of metastatic samples.

There is an important dichotomy with respect to the pro- or anti-tumorigenic effects of PGC1 $\alpha$ in different cancer types ${ }^{20,24}$ and even within a cancer type ${ }^{19,25}$, so is the impact of PGC1 $\alpha$ on metastasis ${ }^{12,26,27}$. Furthermore, the regulatory effect of PGC1 $\alpha$ on the growth and metastasis of a cancer type also shows a discrepancy. For instance, PGC1 $\alpha$ promotes prostate cancer growth but suppresses metastasis ${ }^{28,29}$. Our study and some other studies show that PGC1 $\alpha$ has no impact on tumor growth but promotes metastasis ${ }^{12}$. The reasons for this heterogeneous response remain vague.

Some studies shows that successfully metastasizing cells undergo reversible metabolic changes from oxidative metabolism to glycolysis in an effort to reduce ROS production $^{30}$. In contrast, it has been reported that mitochondrial respiration fuels tumor cell invasion ${ }^{31,32}$. Given the diverse outcomes of PGC1 $\alpha$ on tumor phenotype, we postulate that it is likely dictated at least in part by the self-control balance of PGC1 $\alpha$ between metabolic process and redox stress. Our data suggest that OXPHOS 
enhancement induced by PGC1 $\alpha$ is accompanied by an increase in ROS levels in CCA cells. As PGC1 $\alpha$ simultaneously upregulates antioxidant scavenging system of cancer cells, the ROS levels in CCA cells reach a higher equilibrium state where ROS is not completely scavenged but below a threshold. To the end, the increased ROS levels are not enough to deteriorate OXPHOS and induce mitochondria-mediated apoptosis but support metastatic dissemination of CCA cells (Fig. 8f). Similarly, in other studies, if PGC1 $\alpha$-positive cells exhibit remarkably increased ROS detoxification capacities, PGC1 $\alpha$ predominantly enhances mitochondrial metabolism and thus facilitates tumor growth ${ }^{19}$. In contrast, if overly high ROS levels that cannot be appropriately counterbalanced by the cellular antioxidant defense system would induce oxidative stress and tumor growth suppression; ${ }^{20}$ in circulating cancer cells, excessive ROS levels would reduce mitochondrial function and thus limit distant metastasis $^{33}$.

\section{Materials and methods}

Tissue samples collection

Between 2007 to 2015, 100 CCA and non-tumor adjacent specimens and 30 normal bile duct tissues were collected during surgery at the First Affiliated Hospital of Harbin Medical University. The histopathology diagnosis was based on the World Health Organization criteria. The clinical classification of tumors was defined according to the International Union against Cancer TNM classification system. Tumor differentiation was assigned according to the Edmondson grading system. Ethical approval was acquired from the Research Ethics Committee of the First Affiliated Hospital of Harbin Medical University with the informed consent of each patient. The detailed clinicopathologic characteristics of all CCA patients are listed in Supplementary Table 1.

\section{Microarray analysis}

RNA was extracted from the HuCCT1 cell line using Trisol (Invitrogen) and submitted to Biolancet Technology, Ltd. (Beijing, China). Gene expression microarray was performed using the Affymetrix GeneChip Human Transcriptome Array 2.0 and analyzed using Affymetrix Transcriptome Analysis Console software. The heatmap was drawn using $\mathrm{R}$ software.

\section{Co-immunoprecipitation assay}

Cells were washed twice with cold phosphate-buffered saline (PBS), scraped off, centrifuged and lysed in $300 \mu \mathrm{l}$ buffer containing a protease inhibitors cocktail (SigmaAldrich). After centrifugation and preclearing of the lysate by incubating with $20 \mu \mathrm{l}$ of protein A/G beads (Santa Cruz Biotechnology, USA) for $1 \mathrm{~h}$ at $4{ }^{\circ} \mathrm{C}, \mathrm{PGC} 1 \alpha$ complexes were precipitated using anti-PGC1 $\alpha$ antibody for $12 \mathrm{~h}$ and protein A/G beads for $2 \mathrm{~h}$. Precipitates were washed four times with lysis buffer, denatured by boiling in loading buffer, and detected by western blotting.

\section{Luciferase activity assay}

The potential NRF1 sites on the human PDHA1 or MPC1 promoter were predicted on JASPAR websites. The candidate NRF1-binding sites on the PDHA1 and MPC1 promoter were cloned in a SV40 promoter driving luciferase reporter plasmid. The plasmid together with a NRF1 expression vector and (or) PGC1 $\alpha$ expression vector or a control plasmid was transfected into 293T cells. After $48 \mathrm{~h}$, luciferase activity was measured on the dual luciferase reporter assay system (Promega). Expression levels of reporter genes were normalized with renilla luciferase activity.

\section{EMSA}

The radiolabeled dsDNA probes encompassing the NRF1-binding site from the PDHA1 promoter or the NRF1-binding site from MPC1 promoter (wild-type probe) were used in the assays, and their mutants (mutant probe), were used to test specific binding of NRF1 to the two sites. The wild-type and mutated unlabeled PDHA1 and MPC1 dsDNA oligonucleotides were used for competition analysis. The oligonucleotides employed in the assays were as follows (mutated nucleotides were underlined): PDHA1/NRF1, 5'-CCACCTTCCCACGCAGGCG CTATCAAGCCC-3' and $3^{\prime}$-GGTGGAAGG-GTGCGTC CGCGATAGTTCGGG-5'; PDHA1/NRF1 mut, 5'-CCA CCTTCCC-ACGGAGTTACTATCAAGCCC-3', 3'-GGT GGAAGGGTGCC̄TCAATGATAG-TTCGGG-5'; MP C1/NRF1, 5'-CGGGCCCCGCCCCCGTGCGCCACCGAGG-GC-3' and $3^{\prime}$-GCCCGGGGCGGGGGCACGCG GTGGCTCCCG-5'; MPC1/NRF1 mut, 5'-CGGG CCCCGCCCCGGTTTACCACCGAGGGC-3' and 3' - GCCCGGGGCGGGGCCAAATGGTGGCTCCCG-5'. A total of $10 \mathrm{ng}$ nuclear extracts of HuCCT1- PGC1 $\alpha$ cells were incubated with $1000 \mathrm{fmol}$ of labeled probes or $100 \mathrm{pmol}$ unlabeled probes, followed by adding gel loading buffer. The mixture was then electrophoresed on a $6.5 \%$ nondenaturing polyacrylamide gel.

\section{ChIP and PCR amplification}

Formaldehyde fixation, cell lysis, and sonication were carried out as previously described ${ }^{34}$. In total, $1 \mu \mathrm{g}$ rabbit anti-NRF1 (abcam; ab34682) or $1 \mu \mathrm{g}$ nonspecific immunoglobulin G (Santa Cruz) were used to immunoprecipitate chromatin. Input and immunoprecipitated DNA were subjected to reversing cross-links and purifying. Then real-time PCR experiments were performed by KangChen Bio-tech, Shanghai, China. Primers flanking the NRF1 site on the PDHA1 promoter: sense, 5'-GCAATCCCACTAGGACACAACA-3'; anti-sense, 
5'-ACACACAGAGACAAAACACCAAAG-3'. Primers flanking the NRF1 site on the MPC1 promoter: sense, 5'-CACAAACTGGCTTGTCTATCCT-3'; anti-sense, 5'-TTCTCAACATTAGTGACCCGC-3'; Primers for GAPDH (negative control): sense, 5'-TACTAGCGG TTTTACGGGCG-3'; anti-sense, 5'-TCGAACAGGA GGAGCAGAGAGCGA-3'. Primers for MEF2A (positive control), sense, 5'-ACCGAGAGGATAATT CAGTCCTG-3'; anti-sense, 5'-ACATCCGCGCACGGATC- $3^{\prime}$. One third of the reaction was electrophoresed on a $5 \%$ polyacrylamide gel, followed by quantification with a PhosphoImager (Molecular Dynamics) and Image Quant software.

\section{LC-MS/MS metabolomics}

The cells were washed twice with PBS and once with saline. After adding $1 \mathrm{ml}$ of a solution of methanol/acetonitrile/water (2:2:1 volume), the cells were scraped off quickly and quenched in liquid nitrogen. Samples were then vortexed and processed by ultrasonication at $4{ }^{\circ} \mathrm{C}$ and incubated $1 \mathrm{~h}$ at $-20^{\circ} \mathrm{C}$ to pellet cell debris and proteins. Samples were subsequently centrifuged at $14,000 \mathrm{rpm}$ for $15 \mathrm{~min}$ at $4{ }^{\circ} \mathrm{C}$ and supernatants were saved. Metabolite extractions were dried by SpeedVac with no heat. Samples were re-suspended by adding $100 \mu \mathrm{l}$ of a solution of acetonitrile/water (1:1 volume) and then separated by hydrophilic interaction chromatography. Some metabolites were targeted in negative ion MRM mode. Metabolomics data analysis was done using Metaboanalyst software.

\section{Animal studies}

Male BALB/c nude mice (5-6 weeks old) were obtained from Beijing Vital River Laboratory Animal Technology Co., Ltd., and housed in a specific pathogen-free environment. All animal experiments were conducted in accordance with standard protocols of the Institutional Animal Care and Use Committee of Harbin Medical University. For xenograft tumor growth, $3 \times 10^{6} \mathrm{CCA}$ cells suspended in $200 \mu \mathrm{l}$ of PBS were injected into the flanks of mice ( $n=10$ /group). Tumor size was measured with Vernier calipers weekly, and volume was calculated using the formula: volume $=$ length $\times(\text { width })^{2} \times 0.52$. The mice were monitored over 4 weeks for tumor formation. To evaluate long-distance lung metastasis, the cells were transfected with a lentivirus containing firefly luciferase, and then $3 \times 10^{6}$ CCA cells suspended in $150 \mu$ of PBS were injected into nude mice ( $n=10$ /group) through the tail vein. D-Luciferin (Gold Biotechnology, Hopkinton, MA) was administered intraperitoneally at $100 \mathrm{mg} / \mathrm{kg}$ body weight, and bioluminescence was detected with a Berthold NIGHTOWL LB983 imaging machine. Mice were killed at 12 weeks, and the lung metastases were confirmed by $H \& E$ staining. To evaluate the peritoneal metastasis, $3 \times 10^{6} \mathrm{CCA}$ cells in $200 \mu \mathrm{l}$ of PBS were injected into the intraperitoneal cavity of $6-8$-week-old male BALB/c mice ( $n=10 /$ group). Mice were imaged at 4 weeks after injection and then killed, and the number of metastatic nodules was counted.

Details on other experimental procedures are described in the Supplementary Methods.

\section{Acknowledgements \\ This work was supported by the China Medical Board (08-894), Outstanding Youth Training Fund from Academician Yu Weihan of Harbin Medical University (2014), Natural Science Foundation of Heilongjiang Province of China (QC2013C094, JJ2018LX0019), and the National Natural Scientific Foundation of China $(81100305,81470876,81502605$, and 81270527).}

\section{Author details}

${ }^{1}$ Department of Hepatic Surgery, The First Affiliated Hospital of Harbin Medical University, Key Laboratory of Hepatosplenic Surgery, Ministry of Education, Harbin, Heilongjiang Province, China. ${ }^{2}$ Key Laboratory of Hepatosplenic

Surgery, Ministry of Education, The First Affiliated Hospital of Harbin Medical University, Harbin, Heilongjiang Province, China

Conflict of interest

The authors declare that they have no conflict of interest.

\section{Publisher's note}

Springer Nature remains neutral with regard to jurisdictional claims in published maps and institutional affiliations.

Supplementary Information accompanies this paper at https://doi.org/ 10.1038/s41419-018-0494-0.

Received: 17 January 2018 Revised: 9 March 2018 Accepted: 12 March 2018 Published online: 27 April 2018

\section{References}

1. Rizvi, S. \& Gores, G. J. Pathogenesis, diagnosis, and management of cholangiocarcinoma. Gastroenterology 145, 1215-1229 (2013).

2. Khan, S. A. et al. Guidelines for the diagnosis and treatment of cholangiocarcinoma: an update. Gut 61, 1657-1669 (2012).

3. Razumilava, N. \& Gores, G. J. Cholangiocarcinoma. Lancet 383, 2168-2179 (2014).

4. Kroemer, G. \& Pouyssegur, J. Tumor cell metabolism: cancer's Achilles' heel. Cancer Cell. 13, 472-482 (2008)

5. Hirschey, M. D. et al. Dysregulated metabolism contributes to oncogenesis. Semin. Cancer Biol. 35, S129-S150 (2015). Suppl.

6. Dang, C. V. Links between metabolism and cancer. Genes Dev. 26, 877-890 (2012).

7. Weinberg, S. E. \& Chandel, N. S. Targeting mitochondria metabolism for cancer therapy. Nat. Chem. Biol. 11, 9-15 (2015).

8. Lin, J., Handschin, C. \& Spiegelman, B. M. Metabolic control through the PGC-1 family of transcription coactivators. Cell Metab. 1, 361-370 (2005).

9. Villena, J. A. New insights into PGC-1 coactivators: redefining their role in the regulation of mitochondrial function and beyond. FEBS J. 282, 647-672 (2015).

10. Murphy, M. P. How mitochondia produce reactive oxygen species. Biochem. J. 417, 1-13 (2009).

11. St-Pierre, J. et al. Suppression of reactive oxygen species and neurodegeneration by the PGC-1 transcriptional coactivators. Cell 127, 397-408 (2006).

12. LeBleu, V. S. et al. PGC-1a mediates mitochondrial biogenesis and oxidative phosphorylation to promote metastasis. Nat. Cell Biol. 16, 992-1003 (2014).

13. Kelly, D. P. \& Scarpulla, R. C. Transcriptional regulatory circuits controlling mitochondrial biogenesis and function. Genes Dev. 18, 357-368 (2004).

14. Chan, D. C. Fusion and fission: interlinked processes critical for mitochondrial health. Annu. Rev. Genet. 46, 265-287 (2012). 
15. Willems, P. H., Rossignol, R., Dieteren, C. E., Murphy, M. P. \& Koopman, W. J. Redox homeostasis and mitochondrial dynamics. Cell Metab. 22, 207-218 (2015).

16. Archer, S. L. Mitochondrial dynamics-mitochondrial fission and fusion in human diseases. N. Engl. J. Med. 369, 2236-2251 (2013).

17. Soriano, F. X. et al. Evidence for a mitochondrial regulatory pathway defined by peroxisome proliferator-activated receptor-gamma coactivator-1 alpha, estrogen-related receptor-alpha, and mitofusin 2. Diabetes 55, 1783-1791 (2006).

18. Bonnet, S. et al. A mitochondria-K + channel axis Is suppressed in cancer and its normalization promotes apoptosis and inhibits cancer growth. Cancer Cell 11, 37-51 (2007).

19. Vazquez, F. et al. PGC1alpha expression defines a subset of human melanoma tumors with increased mitochondrial capacity and resistance to oxidative stress. Cancer Cell 23, 287-301 (2013).

20. LaGory, E. L. et al. Suppression of PGC-1a is critical for reprogramming oxidative metabolism in renal cell carcinoma. Cell Rep. 12, 116-127 (2015).

21. Alderton, G. K. Metastasis: metabolic reprogramming in disseminated cells. Nat. Rev. Cancer 14, 703 (2014).

22. Weber, G. F. Metabolism in cancer metastasis. Int. J. Cancer 138, 2061-2066 (2015).

23. Doppler, H. \& Storz, P. Differences in metabolic programming define the site of breast cancer cell metastasis. Cell Metab. 22, 536-537 (2015).

24. Bhalla, K et al. PGC1a promotes tumor growth by inducing gene expression programs supporting lipogenesis. Cancer Res. 71, 6888-6898 (2011).
25. Haq, R. et al. Oncogenic BRAF regulates oxidative metabolism via PGC1alpha and MITF. Cancer Cell 23, 302-315 (2013).

26. Liu, W. et al. Metastasis suppressor KISS1 seems to reverse the Warburg effect by enhancing mitochondrial biogenesis. Cancer Res. 74, 954-963 (2014).

27. Luo, C. et al. A PGCla-mediated transcriptional axis suppresses melanoma metastasis. Nature 537, 422-426 (2016).

28. Tennakoon, J. B. et al. Androgens regulate prostate cancer cell growth via an AMPK-PGC-1a-mediated metabolic switch. Oncogene 33, 5251-5261 (2013).

29. Torrano, V. et al. The metabolic co-regulator PGC1alpha suppresses prostate cancer metastasis. Nat. Cell Biol. 18, 645-656 (2016).

30. Lu, J., Tan, M. \& Cai, Q. The Warburg effect in tumor progression: mitochondria oxidative metabolism as an anti-metastasis mechanism. Cancer Lett. 356 156-164 (2015).

31. Caino, M. C. et al. PI3K therapy reprograms mitochondrial trafficking to fuel tumor cell invasion. Proc. Natl. Acad. Sci. USA 112, 8638-8643 (2015).

32. Caneba, C. A., Bellance, N., Yang, L., Pabst, L. \& Nagrath, D. Pyruvate uptake is increased in highly invasive ovarian cancer cells under anoikis conditions for anaplerosis, mitochondrial function, and migration. Am. J. Physiol. Endocrinol. Metab. 303, E1036-E1052 (2012).

33. Piskounova, E. et al. Oxidative stress inhibits distant metastasis by human melanoma cells. Nature 527, 186-191 (2015).

34. Song, R. et al. Reciprocal activation between ATPase inhibitory factor 1 and NF-kappaB drives hepatocellular carcinoma angiogenesis and metastasis. Hepatology 60, 1659-1673 (2014). 